\title{
HMGB1 Is a Therapeutic Target and Biomarker in Diazepam-Refractory Status Epilepticus with Wide Time Window
}

\author{
Junli Zhao ${ }^{1}$ - Yang Zheng ${ }^{2} \cdot$ Keyue Liu ${ }^{3}$. Junzi Chen ${ }^{4} \cdot$ Nanxi Lai ${ }^{1} \cdot$ Fan Fei ${ }^{1} \cdot$ Jiaying Shi ${ }^{1} \cdot$ Cenglin Xu ${ }^{1}$. \\ Shuang Wang ${ }^{2} \cdot$ Masahiro Nishibori ${ }^{3} \cdot$ Yi Wang $^{1,2} \cdot$ Zhong Chen $^{1,2}$ (D)
}

Published online: 4 December 2019

(C) The American Society for Experimental NeuroTherapeutics, Inc. 2019

\begin{abstract}
Status epilepticus (SE), a life-threatening neurologic emergency, is often poorly controlled by the current pharmacological therapeutics, which are limited to a narrow time window. Here, we investigated the proinflammatory cytokine high mobility group box-1 (HMGB1) as a candidate therapeutic target for diazepam (DZP)-refractory SE. We found that HMGB1 was upregulated and translocated rapidly during refractory SE period. Exogenous HMGB1 was sufficient to directly induce DZPrefractory SE in nonrefractory SE. Neutralization of HMGB1 with an anti-HMGB1 monoclonal antibody decreased the incidence of SE and alleviated the severity of seizure activity in DZP-refractory SE, which was mediated by a Toll-like receptor 4 (TLR4)dependent pathway. Importantly, anti-HMGB1 mAb reversed DZP-refractory SE with a wide time window, extending the therapeutic window from 30 to $180 \mathrm{~min}$. Furthermore, we found the upregulation of plasma HMGB1 level is closely correlated with the therapeutic response of anti-HMGB1 mAb in DZP-refractory SE. All these results indicated that HMGB1 is a potential therapeutic target and a useful predictive biomarker in DZP-refractory SE.
\end{abstract}

Keywords Status epilepticus $\cdot$ HMGB1 $\cdot$ diazepam $\cdot$ anti-HMGB1 monoclonal antibody $\cdot$ time window $\cdot$ biomarker

\section{Introduction}

Status epilepticus (SE) is a neurologic emergency characterized by continuous seizures longer than 5 min or seizures occurring close together without full recovery between seizures $[1,2]$. Current SE treatment protocols suggest a timely progression of treatment [3-5]. The International League

Junli Zhao, Yang Zheng and Keyue Liu contributed equally to this work.

Yi Wang

wang-yi@zju.edu.cn

$\triangle$ Zhong Chen

chenzhong@zju.edu.cn

1 Institute of Pharmacology \& Toxicology, Key Laboratory of Medical Neurobiology of the Ministry of Health of China, College of Pharmaceutical Sciences, Zhejiang University, Hangzhou, China

2 Epilepsy Center, Department of Neurology, Second Affiliated Hospital, School of Medicine, Zhejiang University, Hangzhou, China

3 Department of Pharmacology, Okayama University Graduate School of Medicine, Dentistry and Pharmaceutical Sciences, Okayama, Japan

4 Hangzhou No. 4 High School, Hangzhou, China
Against Epilepsy (ILAE) task force proposes a conceptual definition of SE with two operational dimensions of 5 and $30 \mathrm{~min}$ [6]. Five minutes indicates the time to start the emergency treatment of SE when benzodiazepines, such as diazepam (DZP), are the preferred initial treatment, whereas benzodiazepines/diazepam-refractory SE develops by 30 min post-seizure [7-9]. Failure to treat within this timeframe may result in long-term consequences, such as neuronal loss, gliosis, cognitive dysfunction, epileptogenesis, or even death [10-12]. The intervention time window is one of the most important variables that influence not only the response of SE to anticonvulsants, but also the subsequent high morbidity and mortality of SE, which approaches 20\% [13]. Currently, there is a paucity of effective treatment strategies to control DZP-refractory SE with minimal side effects and complications [14-16]. Thus, the development of alternative safe and effective targets or drugs for controlling DZP-refractory SE remains an urgent unmet clinical need.

Neuroinflammation is an important factor in many types of epilepsy, including SE [17-20]. As an important cytokine, high mobility group box-1 (HMGB1) is actively expressed in epileptic tissues in both animal models and in human patients [21-23]. It was reported that HMGB1 that binds to toll- 
like receptor 4 (TLR4) or receptor for advanced glycation endproducts (RAGE), which can rapidly activate proinflammatory signaling pathways, disrupting the blood-brain barrier (BBB), and heightening the severity of seizures [24-26]. Our previous studies have demonstrated the potential therapeutic role of anti-HMGB1 monoclonal antibody (mAb) in animal acute and chronic spontaneous epilepsy models and human drug-resistant epileptic tissue, as well as its diseasemodifying effect on epileptogenesis [27, 28]. Recently, emerging data from human and animal models also suggest the increased levels of HMGB1 in blood and cerebrospinal fluid are associated with negative therapeutic outcome in many neurological diseases, ranging from traumatic brain injury to epilepsy $[29,30]$. These results indicate that HMGB1 is a key molecular mechanism underlying not only the pathogenesis of epilepsy but also the therapeutic effect in epilepsy. However, whether HMGB1 contributes to SE, especially DZP-refractory SE, is still not fully understood. In the present study, using a kainic acid (KA) induced refractory SE model, we demonstrated that HMGB1 is rapidly expressed and translocated from the nuclei to the cytoplasm during the refractory SE period. Exogenous HMGB1 directly induced DZP-refractory SE whereas neutralization of HMGB1 by anti-HMGB1 mAb reversed DZP-refractory SE with a broad time window. The therapeutic response of anti-HMGB1 mAb to refractory SE was correlated with plasma levels of HMGB1. These results suggest that HMGB1 may be a therapeutic target and a useful predictive therapeutic biomarker in DZP-refractory SE.

\section{Materials and Methods}

\section{Animals}

Male wild-type mice (C57BL/6J) and Tlr $4^{-/}$mice (C57BL/ $10 \mathrm{ScNj}$, Jackson Laboratory: NO.003752) used in this study were 2-4 months old and reared as previously described [27]. All mice were maintained in cages with a 12-h light/dark cycle and behavioral experiments were conducted between 9:00 and 17:00. All experimental procedures performed complied with the Zhejiang University Animal Experimentation Committee and were in compliance with the National Institutes of Health Guide for the Care and Use of Laboratory Animals.

\section{Reagents}

The anti-HMGB1 $\mathrm{mAb}$ was produced as described previously [31] and dissolved in phosphate-buffered saline at a concentration of $2 \mathrm{mg} / \mathrm{ml}$ and stored at $-80{ }^{\circ} \mathrm{C}$ until use. DisulfideHMGB1 (HM-121) was purchased from HMGBiotech. KA (K0250) was purchased from Sigma-Aldrich. DZP (H12020957) was purchased from Tianjin Kingyork Group
Co., LTD. Anti-HMGB1 $\mathrm{mAb}(5 \mathrm{mg} / \mathrm{kg}$ or $10 \mathrm{mg} / \mathrm{kg})$ and $\mathrm{KA}(0.2 \mu \mathrm{g}$ in $0.4 \mu \mathrm{l})$ were dissolved in normal saline to a working concentration before being injected into mice. AntiHMGB1 $\mathrm{mAb}$ and DZP $(10 \mathrm{mg} / \mathrm{kg})$ were administered by i.p. injection. Disulfide-HMGB1 (1 or $3 \mu \mathrm{g})$ was injected into the hippocampus 15 min before KA injection.

\section{Surgery and KA Microinfusion}

The surgery and KA-induced seizures model paradigm was performed as in our previous studies [32, 33]. Briefly, adult mice were anesthetized with sodium pentobarbital $(50 \mathrm{mg} / \mathrm{kg}$, i.p.) and placed in a stereotaxic apparatus (512600, Stoelting). A guide cannula (62004, RWD Life Science) and a bipolarelectrode (791500, A.M. Systems) were stereotaxically implanted into the right hippocampus CA1 (AP $-2.0 \mathrm{~mm}$; ML $-1.3 \mathrm{~mm} ; \mathrm{V}-1.2 \mathrm{~mm}$ ) and CA3 (AP - $2.9 \mathrm{~mm}$; ML -3.0 $\mathrm{mm} ; \mathrm{V}-3.0 \mathrm{~mm}$ ) respectively, based on the mouse brain atlas (Fig. 1a) [34]. After 1-week recovery, an infusion needle (62204, RWD Life Science) was inserted into CA1 through the guide cannula to a depth of $1.7 \mathrm{~mm}$ at a rate of $0.2 \mu 1 / \mathrm{min}$ for KA or/and Disulfide-HMGB1 injection. Following the completion of the infusion, the needle was left in the CA1 for an additional $2 \mathrm{~min}$ to limit reflux. Subsequently, mice were placed into polyvinyl chloride boxes immediately for EEG monitoring with the LabChart system (AD Instruments). The place of the electrode and cannula implantation in all the mice was verified after behavioral tests.

\section{Status Epilepticus and EEG Monitoring}

EEG monitoring was initiated 10 min before hippocampal KA infusion to record the baseline. At the initial stage after KA infusion, there are several changes in EEG, which consist of initial stage of discrete seizures followed by continuous seizures $[35,36]$. The emergence of SE was typically evident in EEG recordings $0-20 \mathrm{~min}$ in mice. SE onset was defined as the appearance of continuous ictal discharge, which was defined as twofold-baseline high amplitude discharges with frequency greater than $3 \mathrm{~Hz}[7,37,38]$. SE was allowed to continue for 10/40/90/180 min before saline/DZP/antiHMGB1 mAb administration. After administration, EEG activity was monitored continuously for at least $3 \mathrm{~h}$. SE was considered terminated when the EEG activity returned to baseline or showed low-frequency irregular spikes without recurrence of seizures in the observation period of $3 \mathrm{~h}$. Seizure events were defined as regular spike clusters with the duration of $\geq 10 \mathrm{~s}$, spike frequency of $\geq 3 \mathrm{~Hz}$, and amplitude at least three times of the baseline EEG similar as previous studies $[39,40]$. The time-course SE-free percentage and EEG power spectrum (30-min interval) of each mouse were analyzed by trained observers blinded to the treatment of mice. 


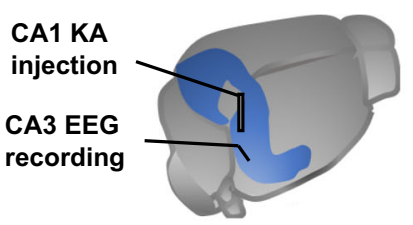

b

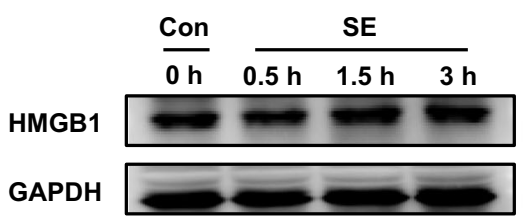

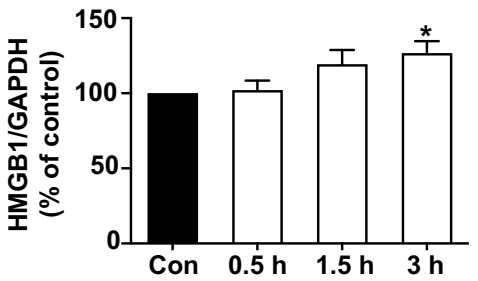

C

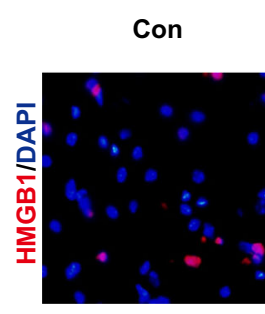

SE-0.5 h

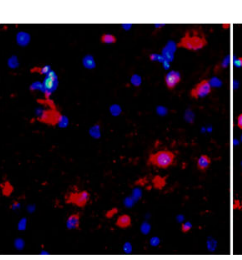

SE-1.5 $\mathrm{h}$

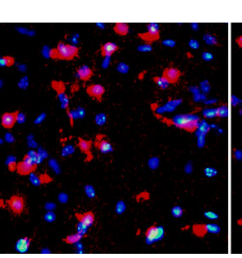

SE-3 h

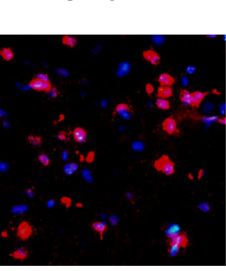

SE-3 h (contra) d

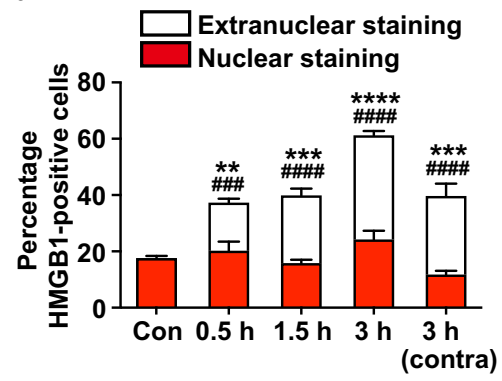

Fig. 1 Prolonged SE upregulates the expression and translocation of HMGB1. a Schematics of sites of KA infusion cannula and EEG recording electrode. $\mathbf{b}$ The expression of HMGB1 protein in the hippocampus after KA-induced SE onset $(n=4)$. $* P<0.05$ versus control, one-way ANOVA followed by Dunnett's test. c Representative images showing HMGB1 translocation in the hippocampus of mice KA-

\section{Immunohistochemistry}

Mice were deeply anesthetized with sodium pentobarbital (100 mg/kg i.p.) and perfused sequentially with normal saline and $4 \%$ paraformaldehyde (PFA). Brains were removed and post-fixed by immersion in $4 \%$ PFA at $4{ }^{\circ} \mathrm{C}$ overnight. After dehydration with $30 \%$ sucrose for $48 \mathrm{~h}$, coronal $20-\mu \mathrm{m}$ sections were cut using a sliding freezing microtome (CRYOSTAR NX50, Thermo Scientific). For immunostaining with HMGB1, slices were incubated at $4{ }^{\circ} \mathrm{C}$ for $2 \mathrm{~h}$ in $5 \%$ donkey serum followed by a $24-\mathrm{h}$ incubation with the primary antibody against HMGB1 (1:400). HMGB1 was visualized by detection with Alexafluor 594 secondary antibodies (712-005153, Molecular Probes) after adding the secondary antibody to HMGB 1 after $2 \mathrm{~h}$. Image slices were mounted with Fluoroshield $^{\mathrm{TM}}$ with DAPI (F6057, Sigma) before images were captured with an Olympus DP70 fluorescence microscope.

\section{Western Blotting}

Hippocampal homogenate $(60 \mu \mathrm{g})$ was resolved with $12.5 \%$ SDS-PAGE, transferred to a nitrocellulose membrane, which was then blocked with 5\% nonfat milk in PBS for $1 \mathrm{~h}$. Then the membranes were incubated with the primary antibodies anti-HMGB1 mAb (1:1000) and anti-GAPDH (1:5000; Kang-chen). After incubation with secondary antibodies goat anti-Rat/Mouse IgG (H + L) HRP (GRT007/GAM007, Multisciences) for $2 \mathrm{~h}$, proteins were visualized by induced after SE onset (red, HMGB1; blue, DAPI). Bar, $50 \mu \mathrm{m}$. d Quantification of HMGB1-positive cells in the hippocampus after KAinduced SE onset $(n=6)$. Total staining: $* * * P<0.001$ and $* * * * P<$

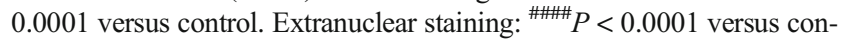
trol; one-way analysis of ANOVA followed by Dunnett's test. All error bars are means \pm s.e.m.

chemiluminescence with an ECL Western Blotting Substrate Kit (61809, Sungky BioTech) according to the manufacturer's instructions. The immunoreactivity of individual bands on western blots was measured by Image-Pro Plus software.

\section{Collection of Blood Sample and Assessment of Total HMGB1}

Blood was collected at two time points in each mouse: 1) baseline prior to KA injection and 2) 90 min after SE onset. Blood $(\sim 40 \mu \mathrm{l})$ was collected from the tail vein and plasma was isolated by centrifugation for $10 \mathrm{~min}$ at $2000 \mathrm{~g}$ at $4{ }^{\circ} \mathrm{C}$ using a refrigerated centrifuge. Following centrifugation, plasma was transferred into a clean polypropylene tube and stored at $-80^{\circ} \mathrm{C}$ until use.

Total HMGB1 level was determined by HMGB1 ELISA kit (ST51011, IBL INTERNATIONAL GMBH) according to the manufacturer's guideline. In brief, thawed samples were centrifuged at $2000 \mathrm{~g}$ for $1 \mathrm{~min}$. Plasma was diluted in the provided diluent buffer at a 1:10 dilution. Samples were then incubated overnight at $37^{\circ} \mathrm{C}$ for $20 \mathrm{~h}$. After incubation, plates were washed 5 times in wash buffer $(400 \mu \mathrm{l} /$ well $)$ and added enzyme conjugate $(100 \mu \mathrm{l} /$ well $)$ for $2 \mathrm{~h}$ at room temperature. Following subsequent washing, $100 \mu$ color solution was added into each well and incubated for $30 \mathrm{~min}$ at room temperature. After incubation, $100 \mu \mathrm{l}$ stop solution was added into each well and the optical density was measured at 450 nm. Results were fitted to the standard curve and unknowns were determined by interpolation. 


\section{Statistical Analysis}

Statistical comparisons were performed using Prism 8 software (GraphPad Software Inc.) and detail information was described in the figure legend. All data were presented as means \pm s.e.m. A two-tailed $P<0.05$ was considered to be statistically significant.

\section{Results}

\section{Prolonged SE Upregulates the Expression and Translocation of HMGB1}

To determine whether SE affects the expression and translocation of HMGB1, we investigated the protein expression and distribution of HMGB1 by western blot and immunohistochemistry in both control and KA-induced SE mice. We found a progressive upregulation of HMGB1 protein in SE mice, with an average of $2.10 \%, 19.30 \%$, and $26.70 \%$ increase in HMGB1 abundance in KA-infusion hippocampus at $0.5 \mathrm{~h}, 1.5$ $\mathrm{h}$, and $3 \mathrm{~h}$ after the SE onset (Fig. 1b). In parallel, we observed a rapid and time-dependent increase in cytoplasmic HMGB1 localization in hippocampal neurons (Fig. 1c). HMGB1 was localized in the nuclei in the hippocampus of control mice, whereas SE increased cytoplasmic HMGB1 signals by 17.05 $\pm 1.439 \%, 24.03 \pm 2.418 \%$, and $36.94 \pm 1.582 \%$ for $0.5 \mathrm{~h}, 1.5$ $\mathrm{h}$, and $3 \mathrm{~h}$ after SE onset, respectively. The contralateral hippocampus of SE-3 $\mathrm{h}$ mice also had significantly increased $(27.91 \pm 4.91 \%)$ cytoplasmic HMGB1 signals. The total HMGB1 signals increased an average of $19.70 \%, 22.28 \%$, $43.60 \%$, and $22.08 \%$, respectively (Fig. 1d), suggesting that increased cytoplasmic HMGB1 staining may be due to the effect of seizures activities. These results indicated that HMGB1 is quickly activated and translocated from the nuclei after SE onset.

\section{HMGB1 Directly Induces DZP-Refractory Phenomenon in Nonrefractory SE}

To further examine whether HMGB1 contributes to DZPrefractory SE, we increased extracellular HMGB1 by intrahippocampal injection of recombinant disulfideHMGB1 15 min prior to KA infusion. Ten minutes post-SE onset (10-min SE), considered as a nonrefractory period, DZP was administered and EEG was continually recorded for $3 \mathrm{~h}$ (Fig. 2a). We found that DZP terminated 10-min SE by an average of $62.5 \%$ and decreased the EEG power throughout the duration of the observation period (Fig. $2 b$ and c). HMGB1 administration (1 and $3 \mu \mathrm{g}$ per mouse) directly induced DZP-refractory phenomenon by decreasing the percentage of SE free to $11.1 \%$ in $1 \mu \mathrm{g}$ HMGB1 administration group and to $0 \%$ in $3 \mu \mathrm{g}$ HMGB1 administration group, respectively (Fig. 2b). Representative power spectra and corresponding seizure activity measured in saline, DZP and/or HMGB1 injected mice is displayed in Fig. $2 \mathrm{~d}$ and e. We observed that HMGB1 administration significantly increased the EEG power of SE (Fig. 2c). These data indicate that HMGB1 directly induces DZP-refractory phenomenon and amplifies seizure activities during SE.

\section{Anti-HMGB1 mAb Controls DZP-Refractory SE via HMGB1-TLR4 Pathway}

Because of the important role of HMGB1 in DZPrefractory SE, we hypothesized that neutralization of upregulated HMGB1 in SE with anti-HMGB1 mAb would control DZP-refractory SE. First, we confirmed that DZP injection $40 \mathrm{~min}$ after $\mathrm{SE}$ onset failed to terminate prolonged SE (Fig. 3a-c), whereas anti-HMGB1 mAb alone increased the percentage of SE free from 0 to $12.5 \%$ (Fig. 3b) and reduced the severity of EEG power (Fig. 3c). Interestingly, co-administration of anti-HMGB1 $\mathrm{mAb}(5 \mathrm{mg} / \mathrm{kg}$ and $10 \mathrm{mg} / \mathrm{kg})$ and DZP increased the percent of SE free from 0 to $37.5 \%$ although also alleviating the severity of seizure activity in the following $3 \mathrm{~h}$ (Fig. $3 b$ and c). Representative EEG power spectra and corresponding raw EEGs are displayed in Fig. 3d and e, respectively. No difference is observed in the therapeutic effect of $5 \mathrm{mg} / \mathrm{kg}$ and $10 \mathrm{mg} / \mathrm{kg}$ anti-HMGB $1 \mathrm{mAb}$, indicating $5 \mathrm{mg} / \mathrm{kg}$ anti-HMGB1 $\mathrm{mAb}$ is sufficient to block HMGB1. In addition, co-administration of anti-HMGB1 $\mathrm{mAb}(5 \mathrm{mg} / \mathrm{kg}$ and $10 \mathrm{mg} / \mathrm{kg})$ and DZP greatly reduced the number of generalized seizures, but had no significant effect on duration of SE and mortality (Fig. 4a). Furthermore, we found that anti-HMGB1 mAb treatment alone was sufficient to reduce neuronal loss in the hippocampus (Fig. 4b).

As HMGB1-TLR4 axis contributes to the generation of epilepsy [26], we next tested whether TLR4 was involved in DZP-refractory SE. We found that DZP alone terminated $37.5 \%$ prolonged SE (40-min SE) in $T l r 4^{-/}$mice compared with those in the saline treatment group (Fig. 5a and b). This percent of SE free in DZP-Tlr $4^{-/}$mice is consistent with the percentage of co-administration of anti-HMGB1 mAb and DZP in WT mice. The corresponding EEG power spectra and raw EEGs also indicated the therapeutic effect of DZP on prolonged SE in $\mathrm{Tlr}^{-/}$mice (Fig. $5 \mathrm{~d}$ and e). In addition, we found the latency between KA infusion and SE onset in $\mathrm{Tlr}^{-/-}$mice $(21.91 \pm 4.603 \mathrm{~min})$ is longer than WT mice $(17.14 \pm 2.513 \mathrm{~min})$ and the EEG power in $T l \mathrm{r} 4^{-/}$mice is also less intense than WT mice (Figs. $3 \mathrm{c}$ and $5 \mathrm{c}$ ), indicating the reduced intrinsic susceptibility to seizure in $\mathrm{Tlr} 4^{-/}$mice. These results suggest that the HMGB1-TLR4 signaling axis contributes to DZP-refractory SE. 


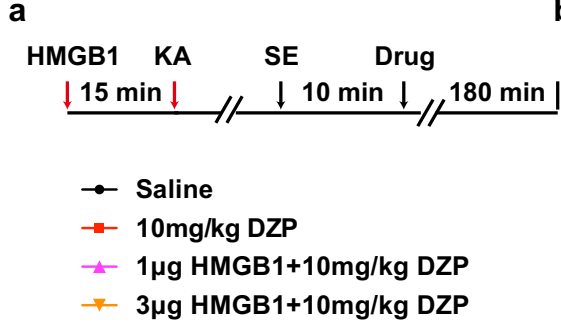

$\checkmark \quad 3 \mu \mathrm{g}$ HMGB1+10mg/kg DZP

d

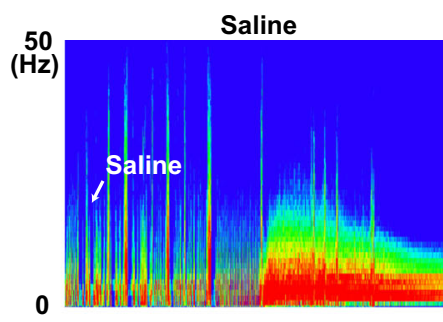

e

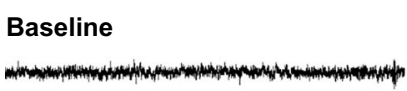

Pre-Saline injection

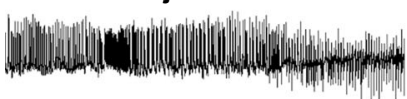

90 min Post-Saline injection

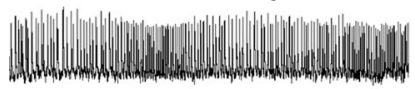

180 min Post-Saline injection

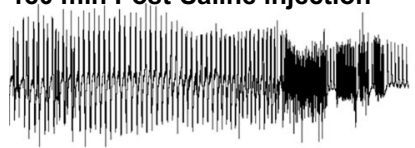

180 min Post-DZP injection
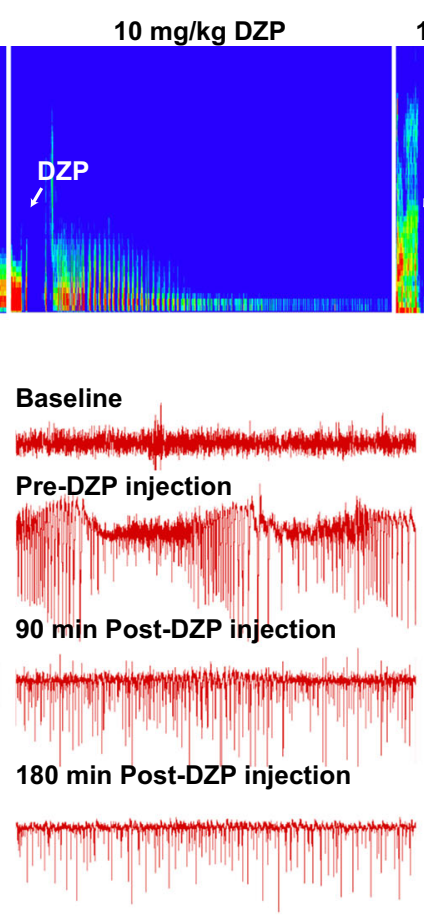

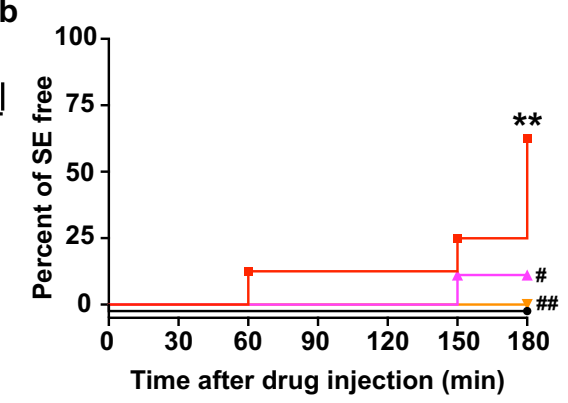

Time after drug injection (min)

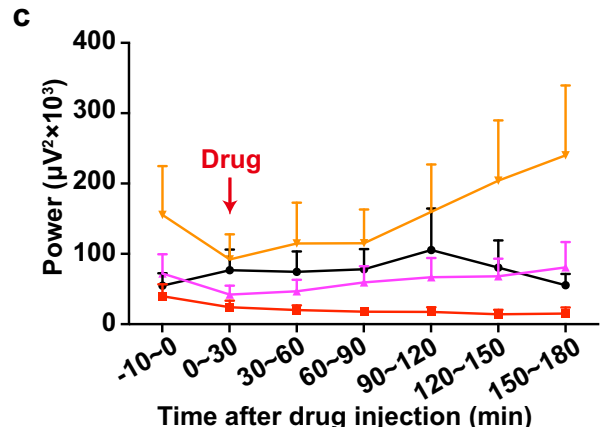

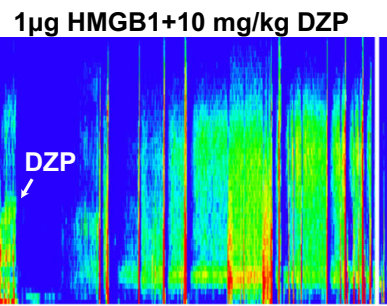

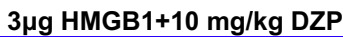

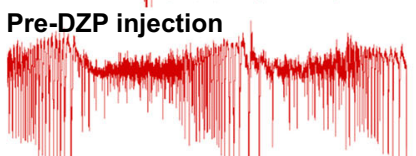

90 min Post-DZP injection

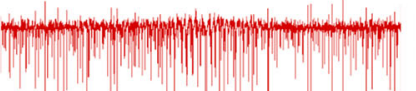

180 min Post-DZP injection

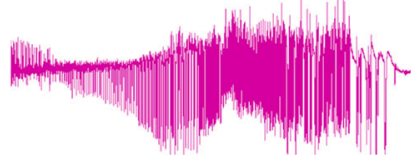

180 min Post-DZP injection

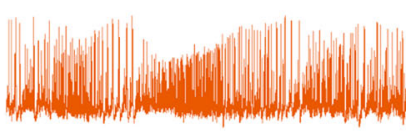

Fig. 2 HMGB1 is sufficient to induce DZP-refractory phenomenon during SE. a Schematic of experimental design: HMGB1 was injected into the hippocampus 15 min before KA infusion. Drug (Saline or DZP) was administered $10 \mathrm{~min}$ after SE onset. $\mathbf{b}$ The time course of SE free after drug administration in different groups $(n=8-9)$. $* * P<0.01$, DZP group versus saline group; ${ }^{\#} P<0.05$, HMGB1 $(1 \mu \mathrm{g})+$ DZP group versus DZP; ${ }^{\# \#} P<0.01$, HMGB1 $(3 \mu \mathrm{g})+$ DZP group versus DZP; Log-rank (Mantel-

\section{Anti-HMGB1 mAb Controls DZP-Refractory SE with a Wide Time Window}

When SE lasts longer (> $60 \mathrm{~min}$ ), it is much more difficult to control, becoming super-refractory SE [12, 41]. To determine whether anti-HMGB1 mAb could control refractory SE that lasts for $>60 \mathrm{~min}$, we administered anti-HMGB1 mAb (5 $\mathrm{mg} / \mathrm{kg}$ ) in 90-min and 180-min SE (Fig. 6a and f). As shown in Fig. 6b and g, SE lasted for $90 \mathrm{~min}$ or $180 \mathrm{~min}$ were DZPrefractory, and co-administration of anti-HMGB1 $\mathrm{mAb}$ and DZP significantly increased the percent of SE free, from 0 to $50 \%$ and 12.5 to $62.5 \%$, respectively. Meanwhile, compared with DZP group, co-administration of anti-HMGB1 $\mathrm{mAb}$ and
Cox) tests were used to compare whole curves. c Quantification of EEG power spectra at each 30-min duration before and after drug administration in different groups $(n=8-9)$. d, e Representative power spectra and corresponding EEGs showing the baseline, pre-drug administration, $90 \mathrm{~min}$ and $180 \mathrm{~min}$ after drug administration. All error bars are means \pm s.e.m.

DZP greatly reduced the severity of EEG power spectra (Fig. 6c, d, h, and j) and the EEG activities (Fig. 6e and j) in 90-min and 180-min SE. In addition, co-administration of antiHMGB1 mAb and DZP greatly shortened the duration of SE in 180-min SE (Fig. 7). These results demonstrated antiHMGB1 mAb could control DZP-refractory SE with a wide time window for intervention.

\section{HMGB1 as a Biomarker in DZP-Refractory SE}

As the results shown in Figs. 3 and 6, even though neutralization of HMGB1 with anti-HMGB1 mAb in DZP-refractory SE significantly increased the percent of SE-free mice, some SE mice 

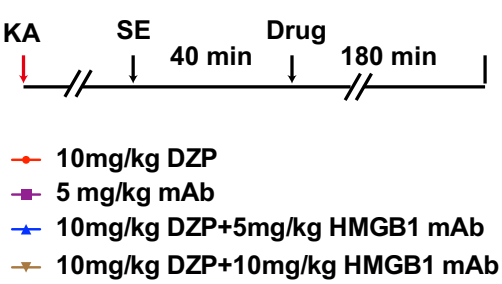

b

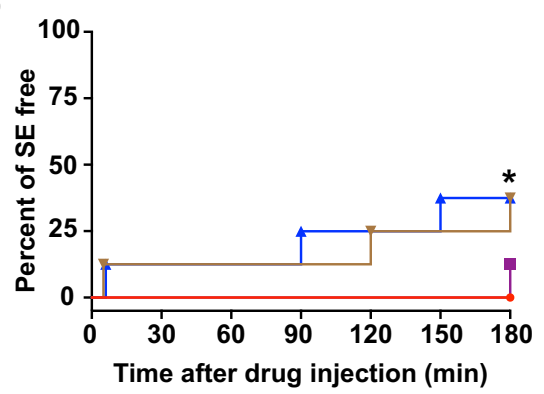

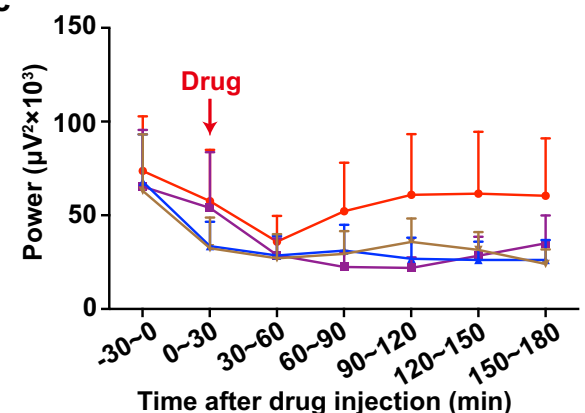

d
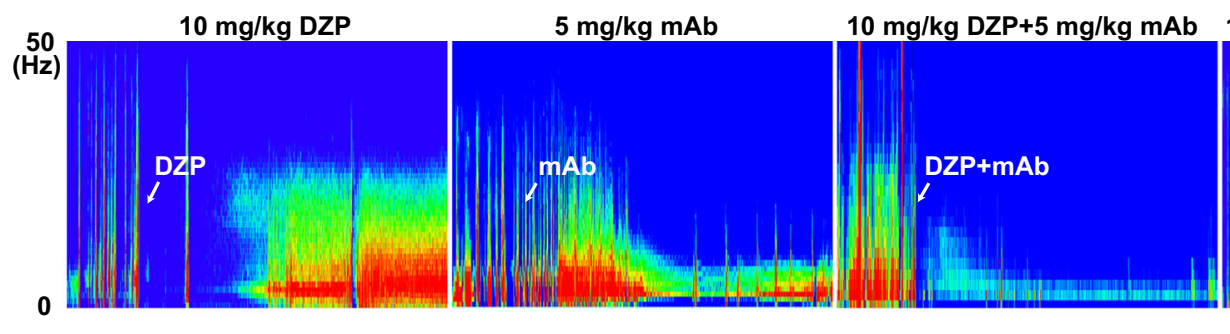

$10 \mathrm{mg} / \mathrm{kg} \mathrm{DZP}+10 \mathrm{mg} / \mathrm{kg} \mathrm{mAb}$

e

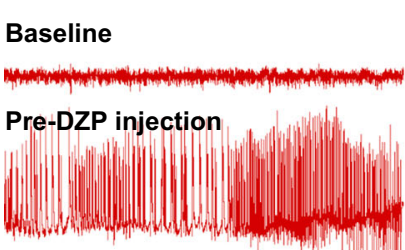

90 min Post-DZP injection

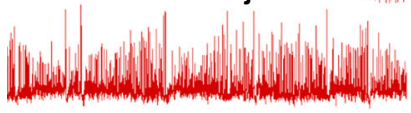

180 min Post-DZP injection

Baseline

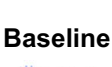

Pre-mAb injection

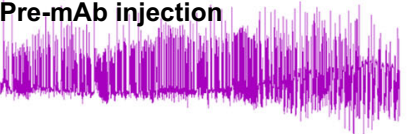

90 min Post-mAb injection
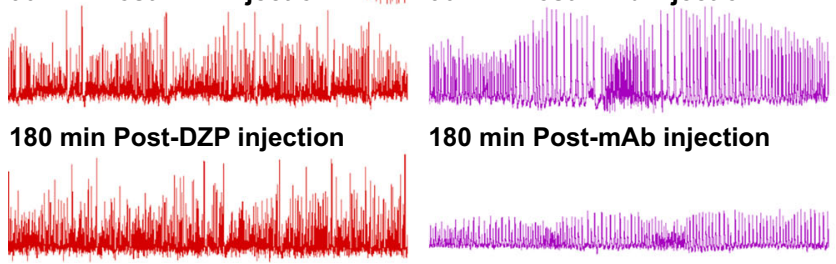

180 min Post-mAb injection

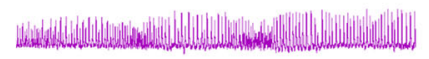

Fig. 3 Anti-HMGB1 mAb increases the percentage of SE free in DZPrefractory SE. a Schematic of experimental design: Drug (DZP or/and anti-HMGB1 $\mathrm{mAb}$ ) was administered $40 \mathrm{~min}$ after SE onset. b The time course of SE free after drug administration in different group $(n=8-9)$. $* P<0.05$, anti-HMGB1 mAb + DZP group versus DZP group, Log-rank (Mantel-Cox) test were used to compare whole curves. c Quantification

do not respond to this treatment. To explain this phenomenon, we collected blood before KA injection and 90 min after SE onset from each mouse to analyze the correlation between the plasma HMGB1 level and the percent of SE free treated by antiHMGB1 mAb. Among all mice treated with the mAb after 90min SE, $50 \%(7 / 14)$ of mice were SE-free (refer as mAbresponse group) whereas 50\% mice were not (referred to as mAb-nonresponse group) (Fig. 8a). Interestingly, quantification of HMGB1 levels showed a striking $66.54 \pm 17.48 \%$ increase in HMGB1 level in mAb-response group, although no significant change ( $-18.55 \pm 20.58 \%)$ of HMGB1 was observed in the mAb-nonresponse group (Fig. 8b). Next, we aimed to see how HMGB1-increased, HMGB1-decreased, and HMGB1-no change mice distributed across the mAb-response and $\mathrm{mAb}$ nonresponse groups. The changed threshold was set as $\pm 20 \%$ of EEG power spectrum at each 30-min duration before and after drug administration in different group $(n=8-9)$. d, e Representative power spectra and corresponding EEGs showing the baseline, pre-drug administration, $90 \mathrm{~min}$ and $180 \mathrm{~min}$ after drug-administration. All error bars are means \pm s.e.m.

for each mouse compared with its own baseline level. We found that all mice in mAb-response group (7/7) had increased HMGB1 level at the timepoint of 90-min SE compared with baseline level, whereas mice in the mAb-nonresponse group showed a mixed changed HMGB1 level, observing $42.86 \%$ (3/7) mice had decreased HMGB1 levels, 28.57\% (2/7) mice increased HMGB1, and $28.57 \%$ (2/7) mice no change (Fig. 8c). Among the SE mice with increased HMGB1, 77.78\% (7/9) mice were successfully SE-free with the treatment of antiHMGB1 mAb (Fig. 8d). These results indicate that plasma HMGB1 levels are closely correlated with the therapeutic response of anti-HMGB1 mAb in DZP-refractory SE, suggesting the upregulation of HMGB1 might be a predictive biomarker not only for DZP-refractory state but also for therapeutic outcome of anti-HMGB1 $\mathrm{mAb}$ in refractory SE. 


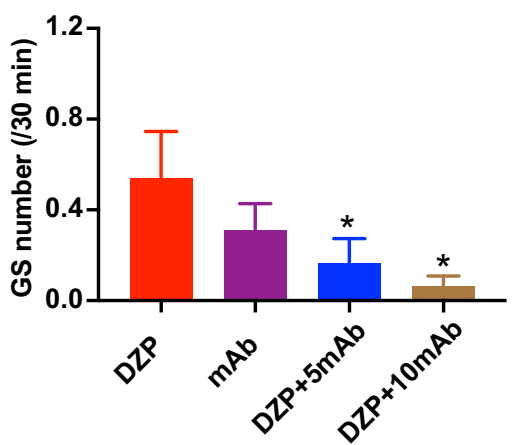

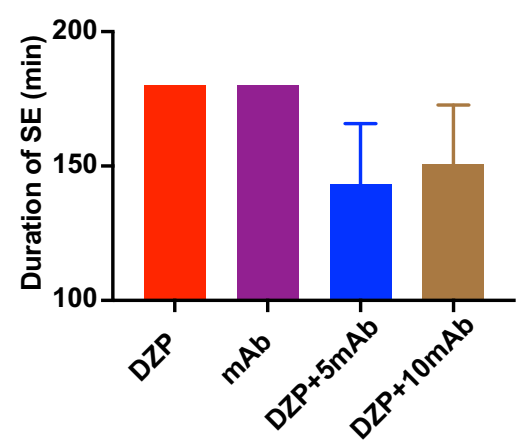

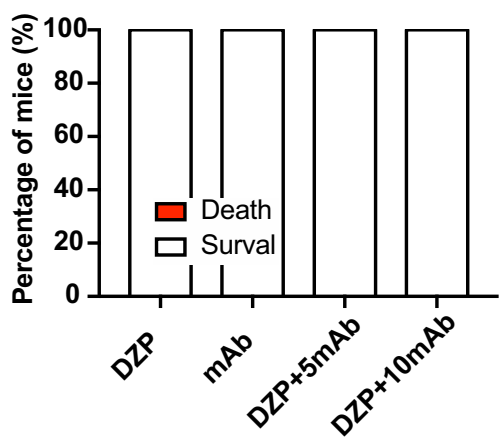

b

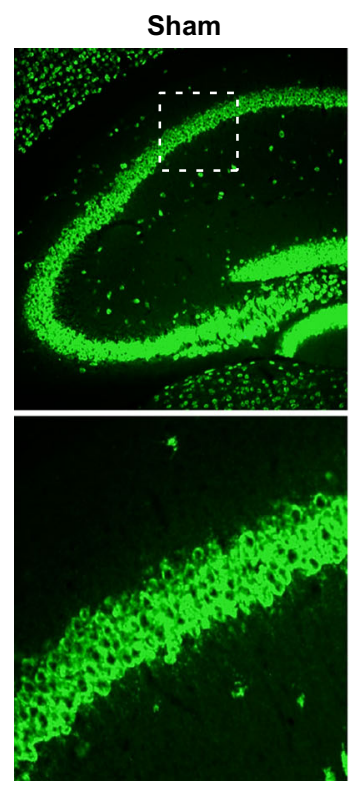

Fig. 4 Anti-HMGB1 mAb reduces the number of generalized seizures during SE and neural loss after SE. a The effect of anti-HMGB1 mAb on the number of generalized seizure, duration of SE and mortality. Drug (DZP or/and anti-HMGB1 $\mathrm{mAb}$ ) was administered 40 min after SE onset

\section{Discussion}

Prolonged SE (> $30 \mathrm{~min}$ ) is poorly controlled by administration of benzodiazepines and often leads to persistent consequences $[42,43]$. Although many potential mechanisms for DZP-refractory SE have been reported, including the internalization of GABAergic receptors [44, 45] and the increase of glutamatergic receptors $[38,46]$, the exact mechanism requires further investigation. Here, we identified an important role of HMGB1-mediated neuroinflammation in DZP-refractory SE. We found that HMGB1 release and translocation from nuclei to cytoplasm as quickly as $30 \mathrm{~min}$ after SE, and increased progressively and cumulatively as SE goes on. Because the duration of SE is one of the most important factors that influence the response of SE to anticonvulsants (DZP-refractory phenomenon), it is highly possible that the increased HMGB1 may also have a correlation with the SE maintenance and contribute to
$\mathrm{SE}-40 \mathrm{~min}$
DZP

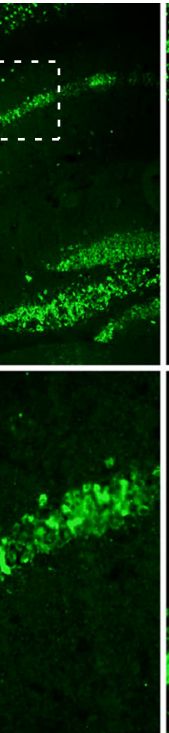

$\mathrm{mAb} 5 \mathrm{mg} / \mathrm{kg}$
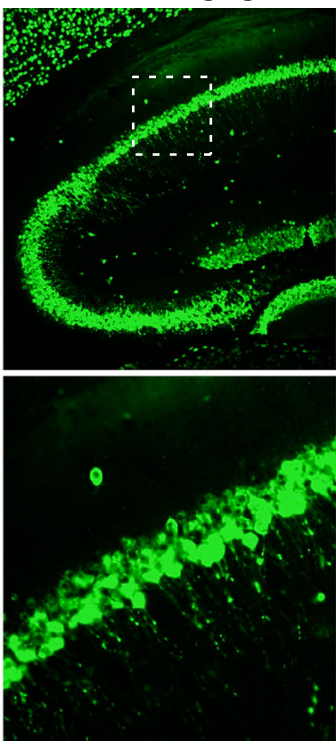

DZP+mAb 5mg/kg
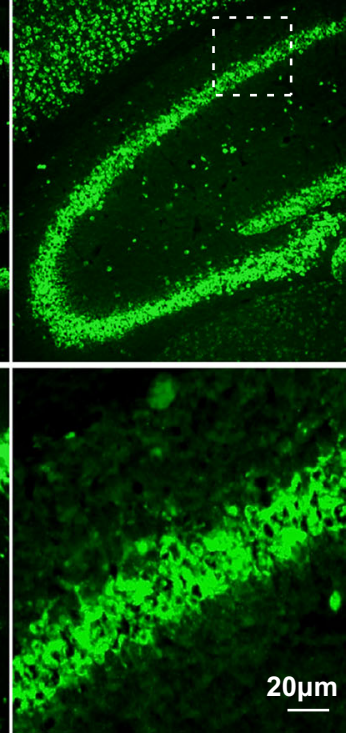

( $n=8-9) . * P<0.05$, compared with DZP group, one-way ANOVA with post hoc Dunnett test was used. b Representative images of NeuN (1:1000, MABN140, Millipore) staining from different groups. All error bars are means \pm s.e.m.

DZP-refractory SE. Injection of exogenous HMGB1 directly induced DZP-refractory SE and aggravated the severity of seizure activity, indicating that HMGB1 may decrease the effectiveness of GABA-mediated inhibitory functions or increase the actions of excitatory neurotransmitters. HMGB1 translocation will result in disruption of $\mathrm{BBB}$, activation, and amplification of a cascade of inflammatory events in the brain [47], which may further potentiate NMDA-mediated calcium $\left(\mathrm{Ca}^{2+}\right)$ influx in excitatory pyramidal neuron $[48,49]$ or may lead to post-translational modifications and downregulated expression of glutamate decarboxylase and glutamate dehydrogenase [50]. Meanwhile, neutralization of excessive HMGB1 with anti-HMGB1 mAb or knockout of its downstream TLR4 receptor apparently increased the percent of SE free in DZP-refractory SE, especially for those prolonged refractory SE, which further indicates that HMGB1 is a key factor in the maintenance of DZP-refractory SE. 


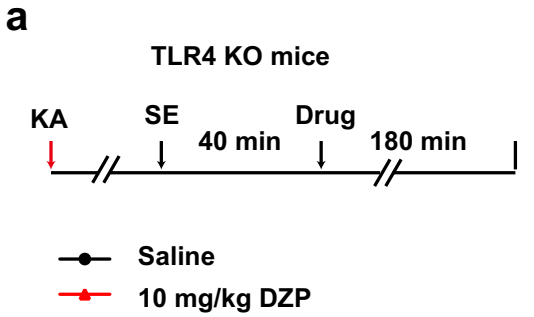

d

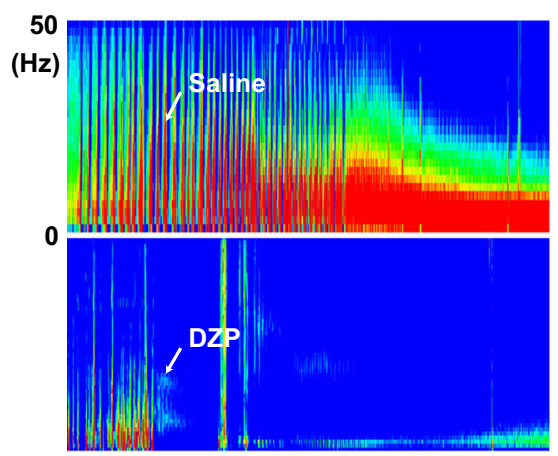

b

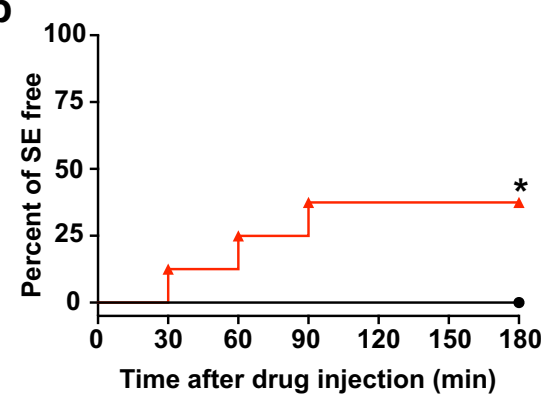

e
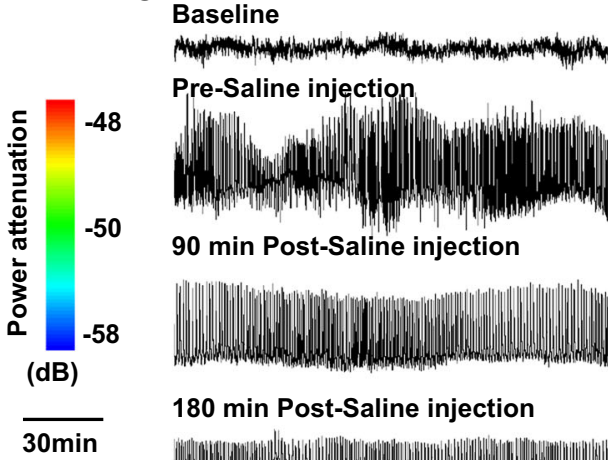

C

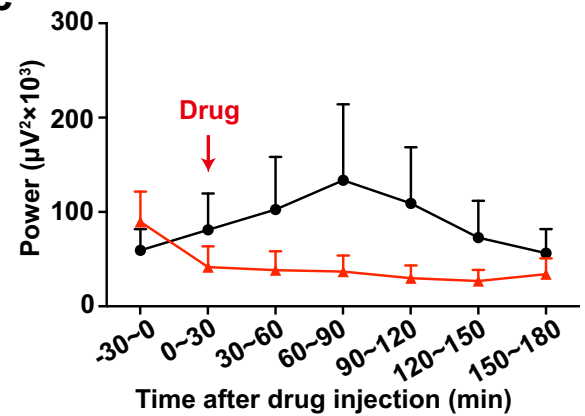

Baseline

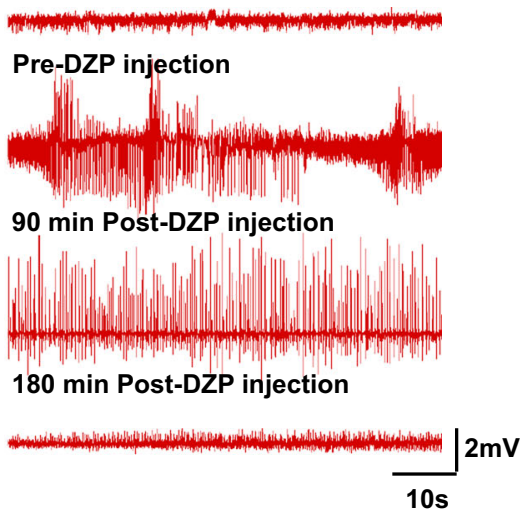

Fig. 5 Increased percentage of SE free in $T L R 4^{-/}$mice with prolonged SE. a Schematic of experimental design: Drug (saline or DZP) was administered $40 \mathrm{~min}$ after SE onset $T l r 4^{-/}$mice. b The time course of SE free in $T l r 4^{-\alpha}$ mice $(n=8-9) . * P<0.05$, DZP group versus saline group, Log-rank (Mantel-Cox) test were used to compare whole curves. $\mathbf{c}$

Administration of anti-HMGB1 $\mathrm{mAb}$ alone did not increase the percentage of SE free mice but consistently reduced the severity of EEGs, which indicates that the mAb could reduce the neural excitability during SE. This is consistent with previous findings that intervention of HMGB1 alone may also have a therapeutic effect on acute and chronic seizure activities in different epileptic models [26, 27]. Particularly, only co-administration of anti-HMGB1 mAb and DZP terminated $37.5 \%$ refractory SE and this treatment produced a synergistic effect greater than their individual effect. As DZP is a positive allosteric modulator of the $\mathrm{GABA}_{\mathrm{A}}$ receptors lasting up to $1 \mathrm{~h}$ [51] whereas anti-HMGB1 $\mathrm{mAb}$ long-termly blocks the HMGB1-TLR4-related inflammatory signaling [27, 52], this synergistic effect may be due to the different molecular targets and pharmacokinetic interaction. This also indicates that multifactorial mechanisms may be involved in DZP-refractory SE, and "cocktail" pharmacological treatment aimed to enhance the degradation of GABAergic transmission combined with anti-HMGB1 mAb would produce synergistic therapeutic effects. Surprisingly, this synergistic therapeutic effect seems to be increasingly potent as the prolonged DZP-refractory SE goes on. Particularly, co-administration of anti-HMGB1 mAb and DZP significantly increased the percent of SE free from 0 to
Quantification of EEG power at each 30-min duration before and after drug administration in different group $(n=8-9)$. d, e Representative power spectra and corresponding EEGs showing the baseline, pre-drug administration, $90 \mathrm{~min}$ and $180 \mathrm{~min}$ after drug-administration. All error bars are means \pm s.e.m.

$50 \%$ when given at $90-\mathrm{min} \mathrm{SE}$, and to $62.5 \%$ when given at 180-min SE. This is consistent with the progressive upregulation and translocation of HMGB1 from 0.5 to $3 \mathrm{~h}$, indicating HMGB1 might play a vital role in SE development, especially in the later maintenance phase. This makes anti-HMGB1 mAb an effective inhibitor on the prolonged DZP-refractory SE with a wide time window.

As we known, previous studies reported that pharmacological blockade of the HMGB1-TLR4 axis with a peptide fragment of HMGB1 (BoxA) and TLR4 antagonist Lps-Rs could produce anti-seizure effects [26]. However, both of above inhibitors have little clinical translational advantage because of the short duration of the therapeutic effect and poor drug-ability. Like other monoclonal antibodies [53, 54], anti-HMGB1 $\mathrm{mAb}$ may have sufficient potency and specificity for therapy and also has good drug-ability with a high therapeutic index, providing ample justification for the further development of this anti-HMGB1 mAb for use in the clinical treatment of epilepsy. We previously demonstrated that even a high-dose of anti-HMGB1 mAb $(25 \mathrm{mg} / \mathrm{kg})$ did not show obvious side effects, including no disrupted physical EEG rhythm and no impaired basic physical functions (body growth rate and thermoregulation) [27]. However, given the role of HMGB1 in transcription, whether are there any other side effects that 


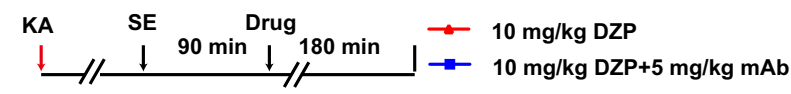

b

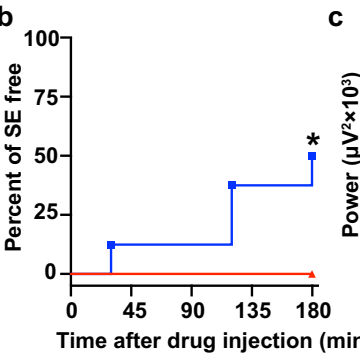

d

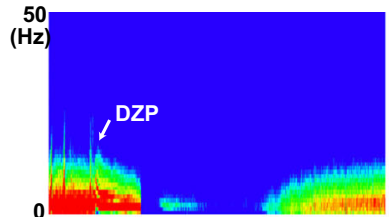

e

Baseline

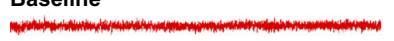

Pre-DZP injection

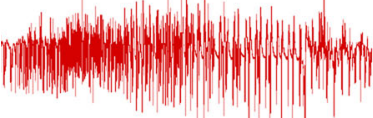

90 min Post-DZP injection

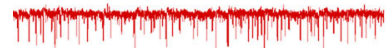

180 min Post-DZP injection

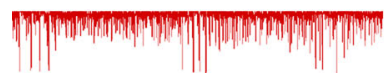

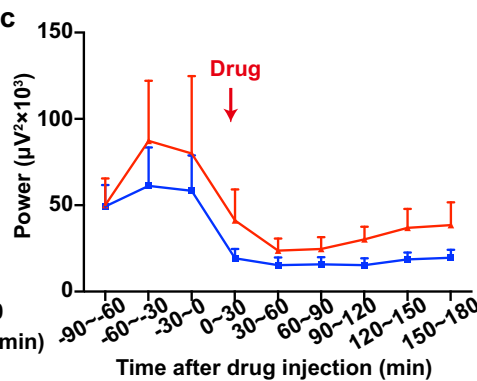

Time after drug injection ( $\mathrm{min}$ )

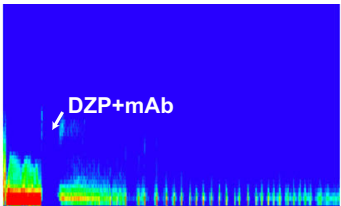

Baseline

Pre-DZP injection

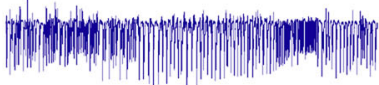

90 min Post-DZP+mAb injection

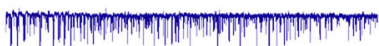
180 min Post-DZP+mAb injection

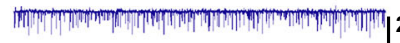

f
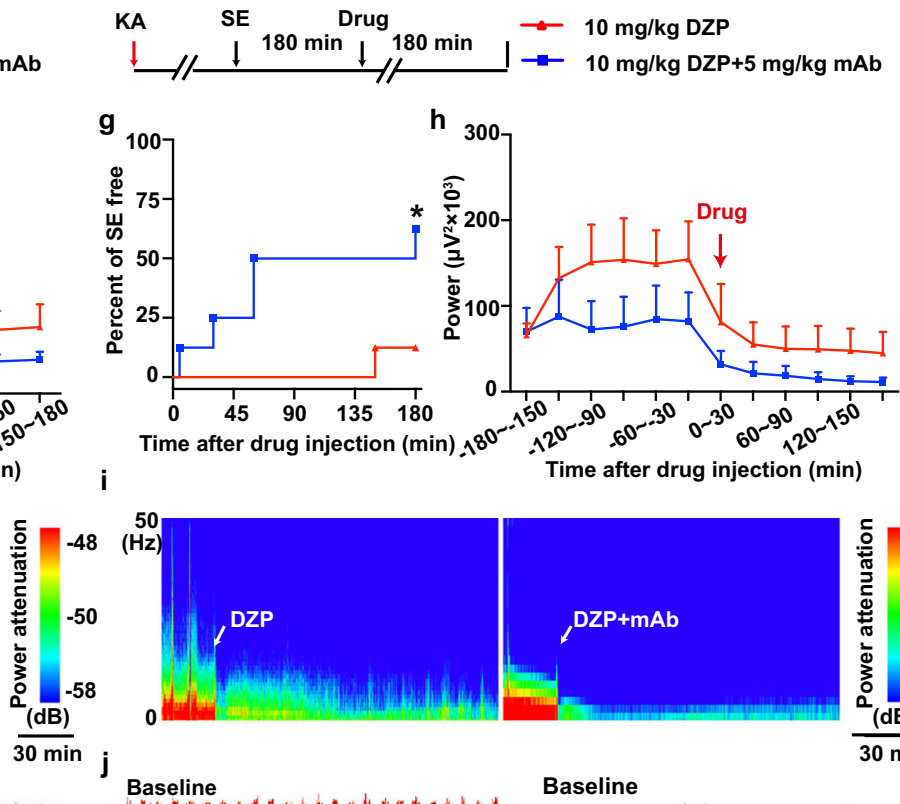

10s

Fig. 6 Anti-HMGB1 mAb controls DZP-refractory SE with a wide time window. a, f Schematic of experimental design: Drug (DZP or/and mAb) was administered $90 \mathrm{~min}$ (a) or $180 \mathrm{~min}$ (f) after SE onset. b, g The time course of SE free after drug administration in different group $(n=8)$. ${ }^{*} P<$ 0.05 versus DZP group, Log-rank (Mantel-Cox) test were used to compare whole curves. c, h Quantification of EEG power at each 30-min

might occur with use of this antibody is still unclear, and the assessment the safety profile of anti-HMGB1 mAb in the clinic was further needed. Meanwhile, BBB disruption commonly occurs in epilepsy [17, 55], including SE condition, and even can be a seizure-promoting effect on epilepsy [56]. Previously,

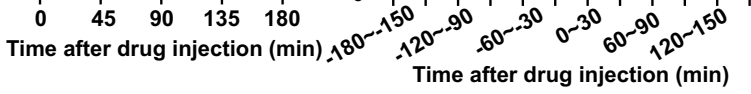

\section{0
$\mathrm{~Hz}$
$\mathrm{~B}$
$\mathrm{P}$}
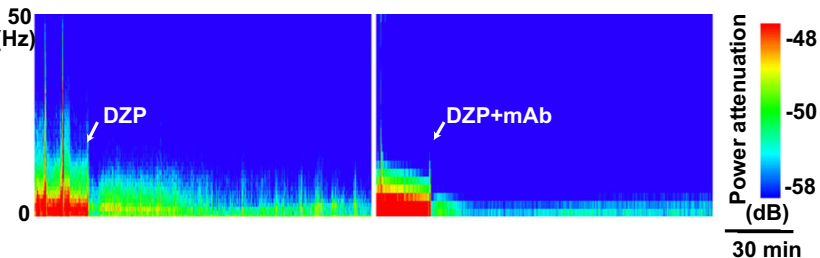

Baseline

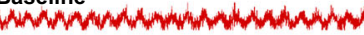

Pre-DZP injection

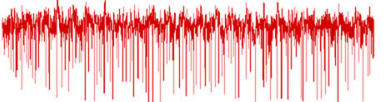

90 min Post-DZP injection

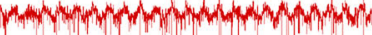

180 min Post-DZP injection

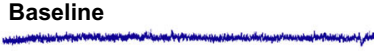

Pre-DZP injection

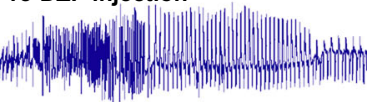

90 min Post-DZP+mAb injection

180 min Post-DZP+mAb injection
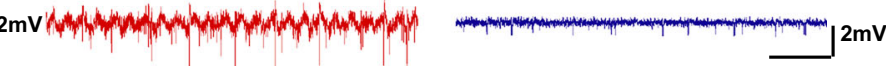

$10 \mathrm{~s}$

duration before and after drug administration in different group $(n=8)$. The EEG power of 30 min was quantified before or after DZP or/and antiHMGB1 mAb was administered. d, e, j, i Representative power spectra and corresponding EEGs showing the baseline, pre-drug administration, $90 \mathrm{~min}$ and $180 \mathrm{~min}$ post drug-administration. All error bars are means \pm s.e.m.

we also detected the BBB leakage in acute seizure models, and found obvious albumin staining after a single MESinduced seizure or after the onset of first EEG seizure in PTZ model [27]. All these suggested that anti-HMGB1 mAb would enter the epileptic brain. To date, human clinical trials

Fig. 7 Anti-HMGB1 mAb shortens duration of SE in prolonged SE. a, b The effect of anti-HMGB1 $\mathrm{mAb}$ on duration of SE. Drug (DZP or/and antiHMGB1 $\mathrm{mAb}$ ) was administered $90 \mathrm{~min}(\mathbf{a})$ or $180 \mathrm{~min}$ (b) after SE onset $(n=8)$. $* P<0.05$, compared with DZP group, nonparametric Mann-Whitney test was used. All error bars are means \pm s.e.m. a

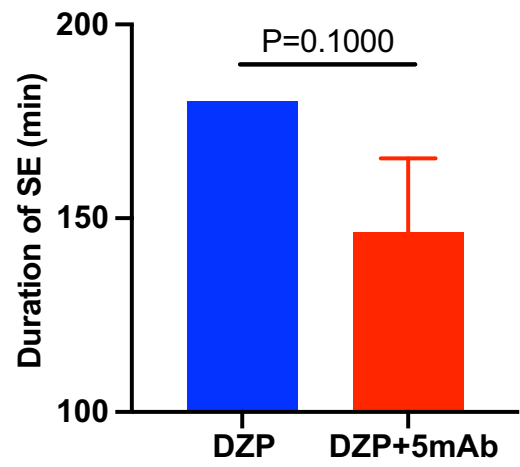

b

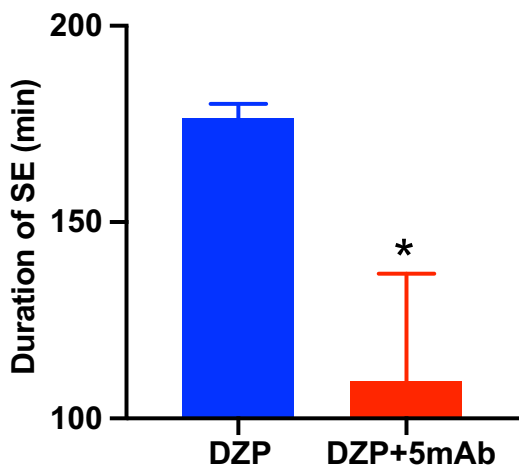


Fig. 8 Relationship between plasma HMGB1 level with the percentage of SE free in mice. a Plasma HMGB1 concentration at baseline and 90-min SE in DZPtreatment mice $(n=14) . * P<0.05$ versus base, paired $t$ test was used. Among all mice treated with the $\mathrm{mAb}$, there were $50 \%(7 / 14)$ mice was SE-free (refer as response group) whereas remaining $50 \%$ mice were not (refer as nonresponse group). b Average change rate of plasma HMGB1 concentration at 90-min SE in DZP-treatment mice $(n=7) .{ }^{* *} P<$ 0.01 versus base, Student's $t$ test was used. Error bars are means \pm s.e.m. $\mathbf{c}$ The distribution of HMGB1-increased, HMGB1-decreased, and HMGB1-no changed mice in $\mathrm{mAb}$-response and $\mathrm{mAb}$ nonresponse groups. The changed threshold was set as $\pm 20 \%$. d The distribution of $\mathrm{mAb}$-response and $\mathrm{mAb}$-nonresponse mice in HMGB1-increased, HMGB1-decreased, and HMGB1-no changed groups

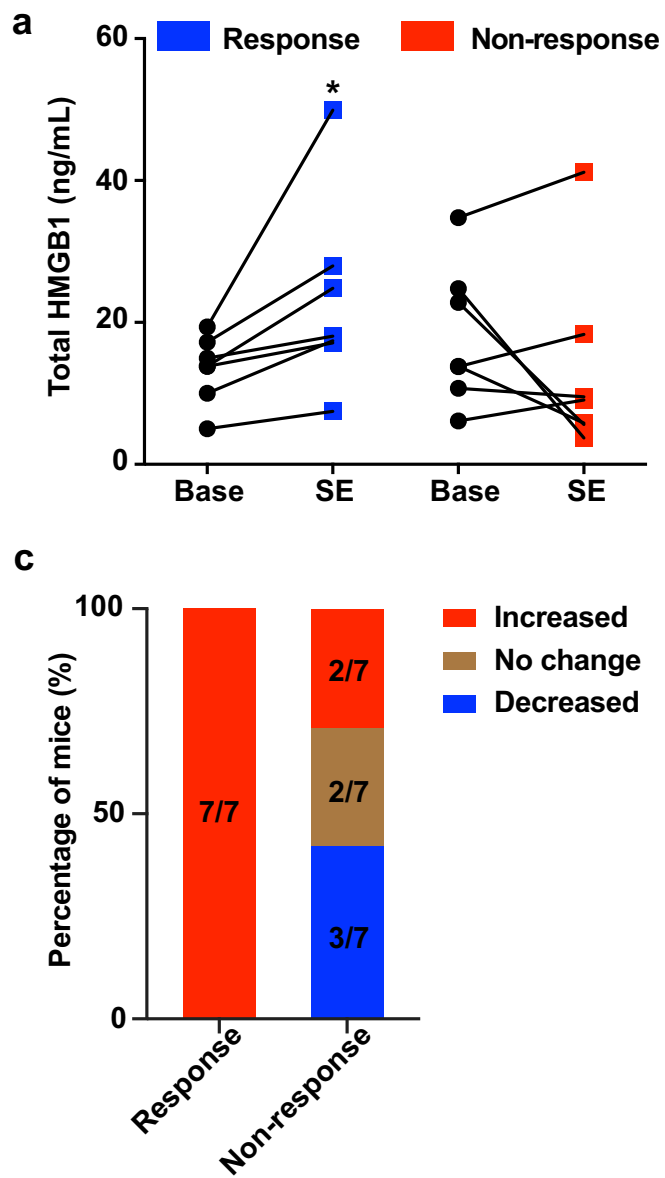

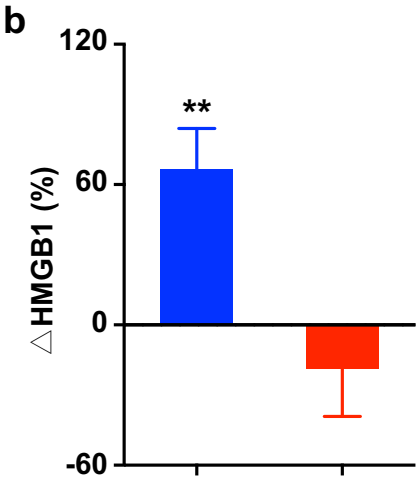

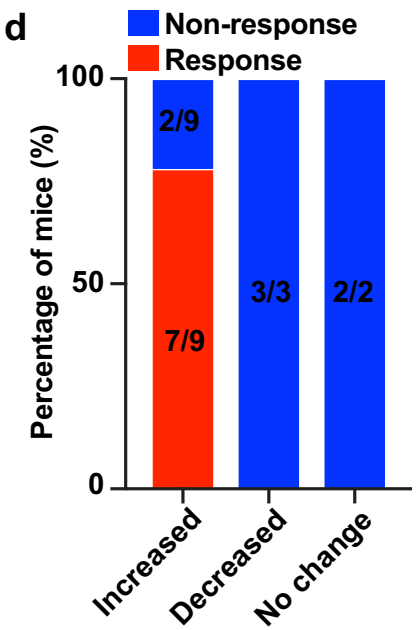

attempting to control prolonged refractory SE mainly use general anesthetics such as midazolam, propofol, or pentobarbitone [57]. However, these treatments require prolonged hospitalization and intensive care and are without no side effect [58]. Thus, anti-HMGB1 mAb holds true promise for use as a safe and effective therapeutic intervention in prolonged DZPrefractory SE with a wide time window.

Although we demonstrated that HMGB1 is a key factor of the overall state of SE, the co-administration of anti-HMGB1 $\mathrm{mAb}$ and DZP did not terminate refractory SE in all mice. This phenomenon reminds us to identify the various characteristic of SE. Interestingly, we found that not every individual mouse that experienced SE showed an increased HMGB1 level, although the averaged HMGB1 level from all mice was indeed upregulated. At the timepoint of 90-min SE (DZP-refractory period), we found that 9/14 mice had increased HMGB1 level compared with their individual baseline level, whereas 3/14 mice had decreased HMGB1 level, and 2/14 mice no change. The change of plasma HMGB1 level between base and SE was related to the percent of SE free treated with anti-HMGB1 mAb. The SE mice which had increased plasma HMGB1 level before anti-HMGB1 mAb administration showed a SE-free percentage as high as $77.78 \%$. Meanwhile, all the mice $(100 \%)$ that response to
anti-HMGB1 mAb treatment had increased HMGB1 level at the timepoint of 90-min SE compared with baseline level. These data strongly suggested that plasma HMGB1 level that can be easily achieved in clinical test is a diagnostic biomarker to differentiate patients who will preferentially response to this treatment. In addition, except for HMGB1, other factors involved in DZP-refractory should be further investigated to control the remaining refractory $\mathrm{SE}$ that are not response to anti-HMGB1 mAb. Taken together, our finding strongly suggested that there may be multifactorial mechanisms underlying DZP-refractory SE, which need individualized treatment scheme. HMGB1 at least may be a valuable predictive biomarker not only for DZP-refractory state but also for therapeutic outcome of anti-HMGB1 mAb in refractory SE.

\section{Conclusion}

The findings in the present study are clinically significant and raise the possibility of using anti-HMGB1 $\mathrm{mAb}$ for the adjunctive treatment of patients with DZP-refractory SE, especially in those with increased plasma HMGB1 levels. Overall, we demonstrate that HMGB1 is a promising therapeutic target and a predictive therapeutic biomarker in prolonged refractory SE. 
Acknowledgments This project was supported by grants from the National Natural Science Foundation of China (81521062, 81630098) and the Fundamental Research Funds for the Central Universities (2019FZA7016). We are very grateful to Dr. Christopher R Donnelly for language editing.

Required Author Forms Disclosure forms provided by the authors are available with the online version of this article.

\section{Compliance with Ethical Standards}

Conflict of Interest The authors declare that they have no conflicts of interest.

\section{References}

1. Betjemann, J.P. and D.H. Lowenstein, Status epilepticus in adults. Lancet Neurol, 2015. 14(6): p. 615-24.

2. Seinfeld, S., H.P. Goodkin, and S. Shinnar, Status Epilepticus. Cold Spring Harbor Perspectives in Medicine, 2016. 6(3).

3. Shorvon, S. and M. Ferlisi, The treatment of super-refractory status epilepticus: a critical review of available therapies and a clinical treatment protocol. Brain, 2011. 134: p. 2802-2818.

4. Shorvon, S., The treatment of status epilepticus. Current Opinion in Neurology, 2011. 24(2): p. 165-170.

5. Verrotti, A. and C. Mazzocchetti, Timely treatment of refractory convulsive status epilepticus. Nature Reviews Neurology, 2018. 14(5): p. 256- + .

6. Trinka, E., H. Cock, D. Hesdorffer, et al., A definition and classification of status epilepticus - Report of the ILAE Task Force on Classification of Status Epilepticus. Epilepsia, 2015. 56(10): p. 1515-1523.

7. Chen, J.W. and C.G. Wasterlain, Status epilepticus: pathophysiology and management in adults. Lancet Neurol, 2006. 5(3): p. 24656.

8. Wasterlain, C.G. and J.W. Chen, Mechanistic and pharmacologic aspects of status epilepticus and its treatment with new antiepileptic drugs. Epilepsia, 2008. 49 Suppl 9: p. 63-73.

9. Wilkes, R. and R.C. Tasker, Pediatric Intensive Care Treatment of Uncontrolled Status Epilepticus. Critical Care Clinics, 2013. 29(2): p. 239- +

10. Brophy, G.M., R. Bell, J. Claassen, et al., Guidelines for the Evaluation and Management of Status Epilepticus. Neurocritical Care, 2012. 17(1): p. 3-23.

11. Power, K.N., A. Gramstad, N.E. Gilhus, K.O. Hufthammer, and B.A. Engelsen, Cognitive dysfunction after generalized tonicclonic status epilepticus in adults. Acta Neurologica Scandinavica, 2018. 137(4): p. 417-424.

12. Sutter, R., S. Semmlack, and P.W. Kaplan, Nonconvulsive status epilepticus in adults - insights into the invisible. Nature Reviews Neurology, 2016. 12(5): p. 281-U62.

13. Neligan, A. and S.D. Shotvon, Frequency and Prognosis of Convulsive Status Epilepticus of Different Causes A Systematic Review. Archives of Neurology, 2010. 67(8): p. 931-940.

14. Sutter, R., S. Marsch, P. Fuhr, P.W. Kaplan, and S. Ruegg, Anesthetic drugs in status epilepticus: risk or rescue? A 6-year cohort study. Neurology, 2014. 82(8): p. 656-64.

15. Santamarina, E., G.M. Gonzalez-Cuevas, A. Sanchez, et al., Prognosis of status epilepticus in patients requiring intravenous anesthetic drugs (a single center experience). Seizure, 2017. 45: p. $74-79$
16. Novy, J., G. Logroscino, and A.O. Rossetti, Refractory status epilepticus: a prospective observational study. Epilepsia, 2010. 51(2): p. 251-6.

17. Gorter, J.A., E.A. van Vliet, and E. Aronica, Status epilepticus, blood-brain barrier disruption, inflammation, and epileptogenesis. Epilepsy Behav, 2015. 49: p. 13-6.

18. Janigro, D., P.H. Iffland, N. Marchi, and T. Granata, A role for inflammation in status epilepticus is revealed by a review of current therapeutic approaches. Epilepsia, 2013. 54: p. 30-32.

19. Vezzani, A. and T. Granata, Brain inflammation in epilepsy: experimental and clinical evidence. Epilepsia, 2005. 46(11): p. 1724-43.

20. Wang, Y. and Z. Chen, An update for epilepsy research and antiepileptic drug development: Toward precise circuit therapy. Pharmacol Ther, 2019.

21. Lotze, M.T. and K.J. Tracey, High-mobility group box 1 protein (HMGB1): nuclear weapon in the immune arsenal. Nat Rev Immunol, 2005. 5(4): p. 331-42.

22. Ravizza, T., G. Terrone, A. Salamone, et al., High Mobility Group Box 1 is a novel pathogenic factor and a mechanistic biomarker for epilepsy. Brain Behavior and Immunity, 2018. 72: p. 14-21.

23. Zurolo, E., A. Iyer, M. Maroso, et al., Activation of toll-like receptor, RAGE and HMGB1 signalling in malformations of cortical development. Brain, 2011. 134: p. 1015-1032.

24. Scaffidi, P., T. Misteli, and M.E. Bianchi, Release of chromatin protein HMGB1 by necrotic cells triggers inflammation. Nature, 2002. 418(6894): p. 191-5.

25. Iori, V., M. Maroso, M. Rizzi, et al., Receptor for Advanced Glycation Endproducts is upregulated in temporal lobe epilepsy and contributes to experimental seizures. Neurobiology of Disease, 2013. 58: p. 102-114.

26. Maroso, M., S. Balosso, T. Ravizza, et al., Toll-like receptor 4 and high-mobility group box-1 are involved in ictogenesis and can be targeted to reduce seizures. Nature Medicine, 2010. 16(4): p. $413-$ U91.

27. Zhao, J., Y. Wang, C. Xu, et al., Therapeutic potential of an antihigh mobility group box-1 monoclonal antibody in epilepsy. Brain Behav Immun, 2017. 64: p. 308-319.

28. Fu, L., K. Liu, H. Wake, et al., Therapeutic effects of anti-HMGBI monoclonal antibody on pilocarpine-induced status epilepticus in mice. Sci Rep, 2017. 7(1): p. 1179.

29. Okuma, Y., K.Y. Liu, H. Wake, et al., Glycyrrhizin inhibits traumatic brain injury by reducing HMGB 1-RAGE interaction. Neuropharmacology, 2014. 85: p. 18-26.

30. Pauletti, A., G. Terrone, T. Shekh-Ahmad, et al., Targeting oxidative stress improves disease outcomes in a rat model of acquired epilepsy. Brain, 2017. 140: p. 1885-1899.

31. Liu, K., S. Mori, H.K. Takahashi, et al., Anti-high mobility group box 1 monoclonal antibody ameliorates brain infarction induced by transient ischemia in rats. FASEB J, 2007. 21(14): p. 3904-16.

32. Wang, Y., J. Liang, C. Xu, et al., Low-frequency stimulation in anterior nucleus of thalamus alleviates kainate-induced chronic epilepsy and modulates the hippocampal EEG rhythm. Exp Neurol, 2016. 276: p. 22-30.

33. Wang, Y., J. Liang, L.Y. Chen, et al., Pharmaco-genetic therapeutics targeting parvalbumin neurons attenuate temporal lobe epilepsy. Neurobiology of Disease, 2018. 117: p. 149-160.

34. George Paxinos, K.B.J.F., The Mouse Brain in Stereotaxic Coordinates. Academic Press, 2001.

35. Treiman, D.M., P.D. Meyers, N.Y. Walton, et al., A comparison of four treatments for generalized convulsive status epilepticus. New England Journal of Medicine, 1998. 339(12): p. 792-798.

36. Treiman, D.M., N.Y. Walton, and C. Kendrick, A Progressive Sequence of Electroencephalographic Changes during Generalized Convulsive Status Epilepticus. Epilepsy Research, 1990. 5(1): p. 49-60. 
37. Xu, Z.H., Y. Wang, A.F. Tao, et al., Interleukin-1 receptor is a target for adjunctive control of diazepam-refractory status epilepticus in mice. Neuroscience, 2016. 328: p. 22-9.

38. Joshi, S., K. Rajasekaran, H.Y. Sun, J. Williamson, and J. Kapur, Enhanced AMPA receptor-mediated neurotransmission on CAl pyramidal neurons during status epilepticus. Neurobiology of Disease, 2017. 103: p. 45-53.

39. Bragin, A., A. Azizyan, J. Almajano, C.L. Wilson, and J. Engel, Analysis of chronic seizure onsets after intrahippocampal kainic acid injection in freely moving rats. Epilepsia, 2005. 46(10): p. 1592-1598.

40. Toyoda, I., S. Fujita, A.K. Thamattoor, and P.S. Buckmaster, Unit Activity of Hippocampal Interneurons before Spontaneous Seizures in an Animal Model of Temporal Lobe Epilepsy. Journal of Neuroscience, 2015. 35(16): p. 6600-6618.

41. Shorvon, S., M. Baulac, H. Cross, et al., Controversial topics in epilepsy. Epilepsia, 2008. 49(7): p. 1277-1284.

42. Hill, C.E., A.O. Parikh, C. Ellis, J.S. Myers, and B. Litt, Timing Is Everything: Where Status Epilepticus Treatment Fails. Annals of Neurology, 2017. 82(2): p. 155-165.

43. Rossetti, A.O., V. Alvarez, J.M. Januel, and B. Burnand, Treatment deviating from guidelines does not influence status epilepticus prognosis. Journal of Neurology, 2013. 260(2): p. 421-428.

44. Naylor, D.E., H.T. Liu, and C.G. Wasterlain, Trafficking of GABA(A) receptors, loss of inhibition, and a mechanism for pharmacoresistance in status epilepticus. Journal of Neuroscience, 2005. 25(34): p. 7724-7733.

45. Kapur, J. and R.L. Macdonald, Rapid seizure-induced reduction of benzodiazepine and $\mathrm{Zn} 2+$ sensitivity of hippocampal dentate granule cell GABA(A) receptors. Journal of Neuroscience, 1997. 17(19): p. $7532-7540$.

46. Niquet, J., R. Baldwin, L. Suchomelova, et al., Benzodiazepinerefractory status epilepticus: pathophysiology and principles of treatment. Countermeasures against Chemical Threats Ii, 2016. 1378: p. 166-173.

47. Zhang, J.Y., H.K. Takahashi, K.Y. Liu, et al., Anti-high Mobility Group Box-1 Monoclonal Antibody Protects the Blood-Brain Barrier From Ischemia-Induced Disruption in Rats. Stroke, 2011. 42(5): p. 1420-1428.

48. Balosso, S., J. Liu, M.E. Bianchi, and A. Vezzani, Disulfide-containing high mobility group box-1 promotes $N$-methyl-D-aspartate receptor function and excitotoxicity by activating Toll-like receptor 4-dependent signaling in hippocampal neurons. Antioxid Redox Signal, 2014. 21(12): p. 1726-40.

49. Pedrazzi, M., M. Averna, B. Sparatore, et al., Potentiation of NMDA receptor-dependent cell responses by extracellular high mobility group box 1 protein. PLoS One, 2012. 7(8): p. e44518.

50. Kaneko, Y., C. Pappas, T. Malapira, et al., Extracellular HMGBI Modulates Glutamate Metabolism Associated with Kainic AcidInduced Epilepsy-Like Hyperactivity in Primary Rat Neural Cells. Cell Physiol Biochem, 2017. 41(3): p. 947-959.

51. Braestrup, C. and R.F. Squires, Specific Benzodiazepine Receptors in Rat-Brain Characterized by High-Affinity [Diazepam-H-3] Binding - (Affinity Binding Diazepam Anxiolytic Activity Brain Membranes Regional Distribution). Proceedings of the National Academy of Sciences of the United States of America, 1977. 74(9): p. 3805-3809.

52. Okuma, Y., K.Y. Liu, H. Wake, et al., Anti-high mobility group box1 antibody therapy for traumatic brain injury. Annals of Neurology, 2012. 72(3): p. 373-384.

53. Hansel, T.T., H. Kropshofer, T. Singer, J.A. Mitchell, and A.J.T George, The safety and side effects of monoclonal antibodies. Nature Reviews Drug Discovery, 2010. 9(4): p. 325-338.

54. Nelson, A.L., E. Dhimolea, and J.M. Reichert, Development trends for human monoclonal antibody therapeutics. Nature Reviews Drug Discovery, 2010. 9(10): p. 767-774.

55. Oby, E. and D. Janigro, The blood-brain barrier and epilepsy. Epilepsia, 2006. 47(11): p. 1761-1774.

56. Marchi, N., L. Angelov, T. Masaryk, et al., Seizure-promoting effect of blood-brain barrier disruption. Epilepsia, 2007. 48(4): p. 732742.

57. Reznik, M.E., K. Berger, and J. Claassen, Comparison of Intravenous Anesthetic Agents for the Treatment of Refractory Status Epilepticus. J Clin Med, 2016. 5(5).

58. Rai, S. and F.W. Drislane, Treatment of Refractory and Superrefractory Status Epilepticus. Neurotherapeutics, 2018.

Publisher's Note Springer Nature remains neutral with regard to jurisdictional claims in published maps and institutional affiliations. 